\title{
Efeito da Ansiedade Competitiva sobre a Tomada de Decisão em Jovens Atletas de Voleibol
}

\author{
Leonardo de Sousa Fortes ${ }^{1, ~ * ~(D), ~ L e n a m a r ~ F i o r e s e ~}{ }^{2}$ (D), José Roberto Andrade \\ Nascimento-Júnior ${ }^{3}$ (D), Sebastião Sousa Almeida ${ }^{4}$ (D), \\ $\&$ Maria Elisa Caputo Ferreira ${ }^{5}$ (D) \\ ${ }^{1}$ Universidade Federal da Paraíba, João Pessoa, PB, Brasil \\ ${ }^{2}$ Universidade Estadual de Maringá, Maringá, PR, Brasil \\ ${ }^{3}$ Universidade Federal do Vale do São Francisco, Petrolina, PE, Brasil \\ ${ }^{4}$ Universidade de São Paulo, Ribeirão Preto, SP, Brasil \\ ${ }^{5}$ Universidade Federal de Juiz de Fora, Juiz de Fora, MG, Brasil
}

\begin{abstract}
RESUMO - O objetivo do estudo foi analisar o efeito da ansiedade competitiva sobre a tomada de decisão de jovens atletas de voleibol. A amostra foi composta por 24 voluntários do sexo masculino (16,06 $\pm 0,65$ anos). Utilizou-se a versão brasileira do Competitite State Anxiety Inventory para avaliar a ansiedade competitiva. A tomada de decisão foi avaliada em situação real de jogo, adotando-se os critérios do Game Performance Assessment Instrument (GPAI). Localizaramse correlações negativas da ansiedade cognitiva e ansiedade somática com o índice de tomada de decisão $(p<0,05)$. Revelaram-se correlações positivas entre a "autoconfiança" e o índice de tomada de decisão $(p<0,05)$. Revelou-se influência da ansiedade cognitiva, somática e autoconfiança no índice de tomada de decisão $(p<0,05)$. Concluiu-se que a ansiedade pode ser considerada um fator interveniente na tomada de decisão.
\end{abstract}

PALAVRAS CHAVE: psicologia do esporte, esporte, atletas

\section{Effect of Competitive Anxiety on Decision-Making in Young Volleyball Athletes}

\begin{abstract}
The aim of this study was to analyze the effect of competitive anxiety on decision-make of young volleyball athletes. The sample was composed of 24 volunteers. We used the Competitive State Anxiety Inventory (CSAI-2R) to evaluate the competitive anxiety. Decision-making was evaluated in real game situation, adopting the criteria of Game Performance Assessment Instrument (GPAI). Negative correlations were found between the decision-making index and both cognitive anxiety and somatic anxiety $(p<0.05)$. In contrast, positive correlation was revealed between self-confidence and the decision-making index $(p<0.05)$. The findings revealed the influence of cognitive anxiety, somatic anxiety and self-confidence in the decision-making index $(p<0.05)$. It was concluded that anxiety can be considered an intervening factor in decision-making of young male volleyball players.
\end{abstract}

KEYWORDS: sport psychology, sport, athletes

* E-mail: leodesousafortes@hotmail.com

n Submetido: 14/10/2016; Revisado: 23/04/2018; Aceito: 28/03/2019. 
O voleibol é um esporte coletivo do tipo intermitente, caracterizado pela imprevisibilidade (Costa et al., 2016; Fortes et al., 2018). A chave para se obter sucesso nessa modalidade concerne na escolha da melhor decisão ("o que fazer"), adotando a técnica correta ("como fazer") em um pequeno espaço de tempo e de forma acurada (Lopes, Magalhães, Diniz, Moreira, \& Albuquerque, 2016). Considera-se o desempenho esportivo um fenômeno multifatorial (Bangsbo, 2015), o qual envolve aspectos técnicos, fisiológicos, biomecânicos, sociais e psicológicos. Dentre os aspectos psicológicos, encontra-se a tomada de decisão.

Entende-se por tomada de decisão uma habilidade do cérebro humano em extrair informações contextuais do cenário visual, as quais são essenciais para o desempenho de alto nível em esportes de imprevisibilidade (Romeas, Gouldner, \& Faubert, 2016). Segundo Afonso, Garganta e Mesquita (2012), a tomada de decisão depende da percepção visual, foco de atenção, antecipação e memória. Além disso, destaca-se que a ação motora também pode influenciar no resultado da tomada de decisão (Lopes et al., 2016), principalmente em atletas amadores. Normalmente o componente cognitivo da tomada de decisão ("o que fazer") de atletas é avaliado por meio de vídeos, os quais simulam situações que acontecem em um jogo (Fortes et al., 2018). Este tipo de análise é conhecido como "conhecimento tático declarativo" (Matias \& Greco, 2011). Todavia, de acordo com Memmert e Harvey (2008), a tomada de decisão envolve tanto o aspecto cognitivo quanto motor. Logo, faz-se necessário utilizar teste que simule situações de jogo e também envolva ações motoras, por exemplo, o Game Performance Assessment Instrument (GPAI), desenvolvido por Oslin, Mitchell e Griffin (1998) e adaptado por Memmert e Harvey (2008).

Estudos revelam que atletas de alto nível demonstram melhor tomada de decisão em comparação aos jovens atletas (Kinrade, Jackson, \& Ashford, 2015; Lopes et al., 2016). Ademais, pesquisadores salientam que os mecanismos cognitivos da tomada de decisão (percepção, atenção, antecipação e memória) são otimizados principalmente durante a adolescência (Araújo et al., 2015). Sendo assim, considera-se importante identificar os fatores intervenientes na tomada de decisão em atletas com idade relativamente precoce. Por um lado, os métodos de treinamento situacionais, adotando-se os "jogos em espaço reduzido", têm indicado efeito positivo na otimização da tomada de decisão de atletas (Davids, Araújo, Correia, \& Vilar, 2013; Romeas et al., 2016). Por outro, parece que o estresse e a ansiedade competitiva podem afetar negativamente a tomada de decisão de atletas (Afonso et al., 2012), embora, de nosso conhecimento, ainda não existam dados científicos que comprovem este apontamento.

A ansiedade competitiva, por sua vez, é considerada um construto multifatorial que diz respeito à disposição de resposta ao estresse e uma tendência de perceber situações estressoras (Fernandes Nunes, Raposo-Vasconcelos, Fernandes, \& Brustad, 2013). De acordo com a Teoria Multidimensional do Estado de Ansiedade Competitiva (Martens, Vealey, \& Burton, 1990), a ansiedade possui três componentes: cognitivo, somático e autoconfiança. A ansiedade cognitiva refere-se aos pensamentos e expectativas negativas relativas ao desempenho. A ansiedade somática concerne nos elementos afetivos e fisiológicos da experiência de ansiedade que afetam diretamente o sistema nervoso autônomo (Fortes et al., 2017). A autoconfiança diz respeito a convicção e o sentimento de capacidade de realizar ações requeridas na competição.

Investigações científicas apontaram relação linear e negativa entre a ansiedade cognitiva e o desempenho físico de atletas (Patel, Omar, \& Terry, 2010; Rathschlag \& Memmert, 2015). Do mesmo modo, estudos demonstraram relação inversamente proporcional entre a ansiedade somática e o desempenho físico de atletas (Fortes et al., 2017; Millet, Groslambert, Barbier, Rouillon, \& Candau, 2005; Silva et al., 2014). Em contrapartida, parece que a autoconfiança revela estreita relação com o desempenho físico de atletas (Fernandes, Nunes, Raposo-Vasconcelos, Fernandes, 2014). Ressalta-se, sobretudo, a inexistência de pesquisas que tenham buscado analisar o efeito da ansiedade competitiva na tomada de decisão de atletas, o que indica o ineditismo do presente estudo.

Do ponto de vista prático, este tipo de investigação poderá revelar o efeito da ansiedade competitiva sobre a tomada de decisão em jovens atletas de voleibol. Neste sentido, os achados poderão ser de extrema importância para os treinadores desta modalidade esportiva. Diante dos apontamentos anteriores, o objetivo do estudo foi analisar o efeito da ansiedade competitiva sobre a tomada de decisão de jovens atletas de voleibol.

Considerando os apontamentos de pesquisadores especialistas na área da tomada de decisão (Afonso et al., 2012; Araújo et al., 2015), formularam-se 3 hipóteses: (H1) quanto maior a intensidade da ansiedade cognitiva, menor será o desempenho na tomada de decisão; $(\mathrm{H} 2)$ quanto maior a intensidade da ansiedade somática, menor será o desempenho na tomada de decisão; e (H3) quanto maior a intensidade da autoconfiança, maior será o desempenho na tomada de decisão. 


\section{MÉTODO}

\section{Participantes}

Trata-se de uma investigação prospectiva com followup de aproximadamente 30 minutos desenvolvida com jovens atletas de voleibol do sexo masculino. A amostra foi selecionada de forma não probabilística, sendo composta por 24 voluntários do sexo masculino com idade entre 15 e 17 anos $(16,06 \pm 0,65$ anos), participantes do campeonato pernambucano de voleibol da categoria sub-17. Os participantes pertenciam a duas equipes vinculadas a Federação Pernambucana de Voleibol.

Os atletas treinavam em média $2 \mathrm{~h}$ por dia, com frequência de cinco vezes por semana. Para serem incluídos na pesquisa, os atletas deveriam: (a) ser atleta de voleibol a pelo menos dois anos; (b) treinar sistematicamente voleibol por pelo menos $8 \mathrm{~h}$ por semana; e (c) estar inscrito no campeonato estadual de voleibol da categoria sub-17, organizado pela Federação Pernambucana de Voleibol.

Após receber informação sobre os procedimentos aos quais seriam submetidos, os participantes assinaram um termo de assentimento. $\mathrm{O}$ treinador dos atletas assinou o termo de consentimento livre e esclarecido (TCLE), concordando com os procedimentos metodológicos da investigação. Os procedimentos adotados neste estudo atenderam às normas da Resolução 466/12 do Conselho Nacional de Saúde para pesquisas em seres humanos. O projeto obteve aprovação do Comitê de Ética e Pesquisa em Seres Humanos da Universidade Federal de Pernambuco (CAE - 46978515.6.0000.5208).

\section{Desenho Experimental}

A versão reduzida em língua portuguesa do Competitite State Anxiety Inventory (CSAI-2R, Fernandes et al., 2013) foi preenchida pelos atletas 30 minutos antes do início de uma partida (quartas de finais) do campeonato pernambucano de voleibol masculino da categoria sub-17. Em seguida, os atletas entraram em quadra para realizarem aquecimento com bola ( $\sim 15$ minutos de duração). Por conseguinte, os atletas foram anunciados individualmente para o público, seguido pelas formalidades que antecedem uma partida de voleibol (cumprimentos aos adversários e a equipe de arbitragem).

Uma câmera CANON ${ }^{\circledR}$ (modelo SX60) foi posicionada em um tripé a 4 metros da quadra de voleibol em frente à mesa dos árbitros. Registrou-se, assim, os 4 sets do jogo de voleibol do campeonato pernambucano sub-17 de voleibol. Todos os procedimentos da investigação estão descritos na Figura 1.

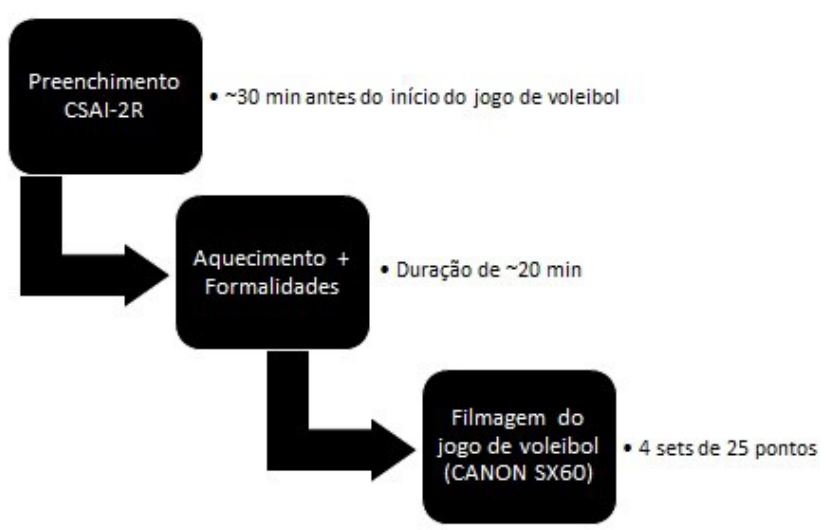

Figura 1. Desenho experimental da investigação

\section{Instrumentos}

Utilizou-se a versão brasileira (Fernandes et al., 2013) do Competitite State Anxiety Inventory (CSAI-2R, Martens et al., 1990) para avaliar a ansiedade competitiva dos atletas. O CSAI-2R é composto por 16 itens que mensuram três subescalas: ansiedade cognitiva, ansiedade somática e autoconfiança. O escore para cada subescala é calculado pela soma das respostas dos itens de cada fator dividida pelo respetivo número de itens. Adotou-se a dimensão de intensidade da CSAI-2R como critério de avaliação da ansiedade competitiva, a qual está disposta em uma escala Likert de 4 pontos, variando de 1 (nada) a 4 (bastante). Quanto maior o escore, maior a magnitude de ansiedade competitiva. Optou-se pela utilização da dimensão frequência da CSAI-2R em razão desta dimensão analisar a magnitude da ansiedade competitiva no momento exato do seu preenchimento. Além disso, cabe destacar que outras investigações científicas também adotaram a dimensão frequência da CSAI-2R (Fortes, Lira, Lima, Almeida, \& Ferreira, 2016; Fernandes et al., 2013). O CSAI-2R foi validado para atletas brasileiros e demonstrou ótimas propriedades psicométricas (Fernandes et al., 2013). Para a presente amostra, se identificou consistência interna (avaliada pelo alpha de Cronbach) de 0,76, 0,81 e 0,79 para as subescalas ansiedade cognitiva, ansiedade somática e autoconfiança, respectivamente.

A tomada de decisão foi avaliada em situação real de jogo. Os participantes disputaram 4 sets de 25 pontos, adotando as regras oficiais do voleibol. Todo o jogo foi filmado com uma câmera $\mathrm{CANON}^{\circledR}$ (modelo SX60). A análise e categorização das ações foram baseadas no GPAI (Memmert \& Harvey, 2008). Os componentes de tomada de decisão no jogo de voleibol propostos por Lopes et al. (2016) foram adotados: saque, recepção e ataque. Para o saque foi 
adotada como ação apropriada as seguintes situações: bola colocada em espaço vulnerável (espaço vazio, jogador menos habilidoso ou ace). Na recepção, considerou-se como ação apropriada as situações que resultaram na bola sendo direcionada as mãos do levantador (e.g., foram considerados todos os tipos de recepções analisadas atualmente no scout). Por fim, para o levantamento, foi adotada como ação apropriada as situações em que a bola foi na mão do atacante (ponta, oposto ou meio).

$\mathrm{O}$ índice de tomada de decisão para cada habilidade técnica (saque, recepção e levantamento) foi calculado de acordo com a fórmula abaixo, considerando as modificações sugeridas por Memmert e Harvey (2008). Cada ação foi analisada por dois especialistas experientes em voleibol e classificada como apropriada ou inapropriada.

$$
\text { Índice de tomada de decisão }=\frac{\left(a_{a}+10\right)}{\left(a_{a}+10\right)+\left(a_{i}+10\right)}
$$

$\mathrm{a}_{\mathrm{a}}=$ ações apropriadas

$\mathrm{a}_{\mathrm{i}}=$ ações inapropriadas

\section{Procedimentos}

A priori, os pesquisadores responsáveis entraram em contato com dois treinadores de duas equipes de voleibol da categoria sub-17 do estado de Pernambuco. Os procedimentos, bem como os objetivos do estudo foram devidamente explicados e foi solicitada a autorização para desenvolver a investigação com os seus atletas.

Em seguida, foi realizada uma reunião com os atletas de voleibol com o intuito de esclarecer sobre todos os procedimentos éticos da investigação. Neste encontro também foi entregue o TCLE ao seu respectivo treinador para consentimento da participação de seus atletas. Todos os atletas assinaram o termo de assentimento, concordando com a sua participação voluntária na investigação.

As coletas dos dados foram realizadas no local de competição (ginásio municipal). Os atletas responderam o CSAI-2R antes do jogo de voleibol. Os jovens atletas receberam a mesma orientação verbal e eventuais dúvidas foram esclarecidas. Em seguida, os atletas disputaram uma partida do campeonato pernambucano de voleibol, a qual foi filmada. Salienta-se que somente 2 profissionais (experientes na área de treinamento em voleibol) analisaram as ações de cada jogador na partida de voleibol.

\section{Análise dos Dados}

Conduziu-se o teste Shapiro Wilk para avaliar a distribuição dos dados. Em razão da não violação paramétrica do índice de tomada de decisão e escore do CSAI-2R, optou-se pela utilização de técnicas paramétricas. Média e desviopadrão foram utilizados para descrever as variáveis (Índice de tomada de decisão, CSAI-2R, regime de treinamento e idade). Utilizou-se a correlação produto-momento de Pearson para relacionar as subescalas do CSAI-2R (ansiedade cognitiva, ansiedade somática e autoconfiança) com o índide de tomada de decisão (saque, recepção e levantamento). Conduziram-se três regressões lineares para analisar: a) a influência das três subescalas da CSAI-2R (ansiedade cognitiva, ansiedade somática e autoconfiança) sobre o índice de tomada de decisão do saque; b) a influência das três subescalas da CSAI-2R (ansiedade cognitiva, ansiedade somática e autoconfiança) sobre o índice de tomada de decisão da recepção e; c) a influência das três subescalas da CSAI-2R (ansiedade cognitiva, ansiedade somática e autoconfiança) sobre o índice de tomada de decisão do levantamento. Todos os dados foram tratados no software SPSS 21.0, adotando-se nível de significância de 5\%.

\section{RESULTADOS}

Os dados descritivos [CSAI-2R, índice de tomada de decisão, regime de treinamento semanal (frequência semanal de treino $\mathrm{x}$ horas de treino diária) e idade] podem ser visualizados na Tabela 1 .
A Tabela 2 indica os valores de correlações entre todas as variáveis da pesquisa. Foram localizadas correlações negativas das subescalas "ansiedade cognitiva" e "ansiedade somática” do CSAI-2R com o índice de tomada de decisão.

Tabela 1

Valores descritivos (média e desvio padrão) das variáveis da pesquisa

\begin{tabular}{lcc}
\hline Variáveis & Média & Desvio Padrão \\
\hline CSAI-2R & 29,53 & 10,26 \\
Índice de Tomada de Decisão (Saque) & 0,55 & 0,07 \\
Índice de Tomada de Decisão (Recepção) & 0,66 & 0,08 \\
Índice de Tomada de Decisão (Levantamento) & 0,72 & 0,06 \\
Regime de treinamento semanal (horas) & 10,28 & 0,84 \\
Idade (anos) & 16,06 & 0,65 \\
\hline
\end{tabular}


Em contrapartida, foram reveladas correlações positivas entre a subescala "autoconfiança" do CSAI-2R e o índice de tomada de decisão.

O modelo de regressão linear que utilizou o índice de tomada de decisão do saque como variável critério pode ser observado na Tabela 3 . Os resultados apontaram influência significante da ansiedade cognitiva $\left(F_{(1,23)}=41,75\right.$; Wilks' Lambda $=0,87 ; R^{2}=0,28 ; p=0,001$ ), inserida no bloco 1 . Identificou-se aumento da magnitude da influência no bloco 2 , quando a ansiedade somática foi inserida no modelo $\left(F_{(2,22)}=61,05 ;\right.$ Wilks' Lambda $\left.=0,93 ; R^{2}=0,51 ; p=0,001\right)$. A autoconfiança, inserida no bloco 3 , revelou aumento da influência sobre o índice de tomada de decisão do saque $\left(F_{(3,21)}=88,60 ;\right.$ Wilks' Lambda=0,96; $\left.R^{2}=0,69 ; p=0,001\right)$.

A regressão linear que adotou o índice de tomada de decisão da recepção como variável dependente pode ser visualizada na Tabela 4. Os achados revelaram influência estatisticamente significante da ansiedade cognitiva $\left(F_{(1,23)}=59,77 ;\right.$ Wilks' Lambda $\left.=0,71 ; R^{2}=0,19 ; p=0,01\right)$. Foi localizado aumento da magnitude da influência no bloco 2 , quando a ansiedade somática foi inserida no modelo $\left(F_{(2,22)}=82,40 ;\right.$ Wilks' Lambda $\left.=0,91 ; R^{2}=0,45 ; p=0,001\right)$. A autoconfiança, inserida no bloco 3 , demonstrou aumento da magnitude da influência sobre o índice de tomada de decisão da recepção $\left(F_{(3,21)}=99,08\right.$; Wilks' Lambda $=0,95$; $\left.R^{2}=0,56 ; p=0,001\right)$.

Por fim, o modelo de regressão linear que utilizou o índice de tomada de decisão do levantamento como variável critério está descrito na Tabela 5 . Os resultados demonstraram influência significante da ansiedade cognitiva $\left(F_{(1,23)}=47,19 ;\right.$ Wilks' Lambda $\left.=0,85 ; R^{2}=0,21 ; p=0,001\right)$, inserida no bloco 1 . Foi revelado aumento da magnitude da influência no bloco 2, quando a ansiedade somática foi inserida no modelo $\left(F_{(2,22)}=67,49\right.$; Wilks' Lambda $=0,89$; $\left.R^{2}=0,39 ; p=0,001\right)$. A autoconfiança, por sua vez, inserida no bloco 3, revelou influência sobre o índice de tomada de decisão do levantamento $\left(F_{(3,21)}=96,33\right.$; Wilks ' Lambda $=0,93$; $\left.R^{2}=0,62 ; p=0,001\right)$.

Tabela 2

Relação entre as subescalas do CSAI-2R e índice de tomada de decisão

\begin{tabular}{lccc}
\hline Subescalas & ITD saque & ITD recepção & ITD levantamento \\
\hline Ansiedade cognitiva & $-0,53^{*}$ & $-0,43^{*}$ & $-0,46^{*}$ \\
Ansiedade somática & $-0,37^{*}$ & $-0,44^{*}$ & $-0,31^{*}$ \\
Autoconfiança & $0,69^{*}$ & $0,33^{*}$ & $0,48^{*}$ \\
\hline
\end{tabular}

Nota. CSAI-2R = Competitive State Anxiety Inventory; ITD = índice de tomada de decisão; ${ }^{\mathrm{p}}<00,05$.

Tabela 3

Regressão linear analisando a influência da ansiedade competitiva (cognitiva, somática e autoconfiança) sobre a variância do indice de tomada de decisão no saque de atletas de voleibol.

\begin{tabular}{lcccccc}
\hline Variável & Bloco & $\boldsymbol{B}$ & $\mathbf{r}$ & $\mathbf{R}^{\mathbf{2}}$ & $\mathbf{R}^{2 *}$ & $\boldsymbol{p}$ valor \\
\hline $\begin{array}{l}\text { Ansiedade } \\
\text { Cognitiva }\end{array}$ & 1 & 0,14 & 0,53 & 0,28 & 0,26 & 0,001 \\
$\begin{array}{l}\text { Ansiedade } \\
\text { Somática }\end{array}$ & 2 & 0,17 & 0,71 & 0,51 & 0,48 & 0,001 \\
Autoconfiança & 3 & 0,19 & 0,83 & 0,69 & 0,66 & 0,001 \\
\hline
\end{tabular}

Nota. $\mathrm{R}^{2 *}=\mathrm{R}^{2}$ ajustado.

Tabela 4

Regressão linear analisando a influência da ansiedade competitiva (cognitiva, somática e autoconfiança) sobre a variância do indice de tomada de decisão na recepção de atletas de voleibol.

\begin{tabular}{lcccccc}
\hline Variável & Bloco & $\boldsymbol{B}$ & $\mathbf{r}$ & $\mathbf{R}^{\mathbf{2}}$ & $\mathbf{R}^{2 *}$ & $\boldsymbol{p}$ valor \\
\hline $\begin{array}{l}\text { Ansiedade } \\
\text { Cognitiva }\end{array}$ & 1 & 0,11 & 0,43 & 0,19 & 0,18 & 0,01 \\
$\begin{array}{l}\text { Ansiedade } \\
\text { Somática }\end{array}$ & 2 & 0,14 & 0,67 & 0,45 & 0,42 & 0,001 \\
Autoconfiança & 3 & 0,18 & 0,75 & 0,56 & 0,54 & 0,001 \\
\hline
\end{tabular}

Nota. $\mathrm{R}^{2 *}=\mathrm{R}^{2}$ ajustado. 
Tabela 5

Regressão linear analisando a influência da ansiedade competitiva (cognitiva, somática e autoconfiança) sobre a variância do índice de tomada de decisão no levantamento de atletas de voleibol.

\begin{tabular}{lcccccc}
\hline Variável & Bloco & $\boldsymbol{B}$ & $\mathbf{r}$ & $\mathbf{R}^{\mathbf{2}}$ & $\mathbf{R}^{\mathbf{2 *}}$ & $\boldsymbol{p}$ valor \\
\hline $\begin{array}{l}\text { Ansiedade } \\
\text { Cognitiva }\end{array}$ & 1 & 0,13 & 0,46 & 0,21 & 0,19 & 0,001 \\
$\begin{array}{l}\text { Ansiedade } \\
\text { Somática }\end{array}$ & 2 & 0,15 & 0,63 & 0,39 & 0,37 & 0,001 \\
Autoconfiança & 3 & 0,18 & 0,79 & 0,62 & 0,61 & 0,001 \\
\hline
\end{tabular}

Nota. $\mathrm{R}^{2 *}=\mathrm{R}^{2}$ ajustado.

\section{DISCUSSÃO}

A investigação teve como objetivo analisar o efeito da ansiedade competitiva sobre a tomada de decisão de jovens atletas de voleibol. Em síntese, os achados do estudo indicaram efeito da ansiedade cognitiva, somática e autoconfiança sobre a tomada de decisão de jovens atletas de voleibol, corroborando as hipóteses do presente estudo.

O modelo teórico de ansiedade competitiva (Martens et al., 1990) diz que a ansiedade cognitiva possui relação linear e negativa com o desempenho esportivo. Em outras palavras, segundo Martens et al. (1990), a ansiedade cognitiva é inversamente proporcional ao desempenho competitivo. Cabe destacar, sobretudo, que o desempenho esportivo é um fenômeno multifatorial (Bangsbo, 2015). Considerando somente o aspecto psicológico do modelo multifatorial de Bangsbo (2015), o desempenho em atletas de esportes de rede/parede, como é o caso do voleibol, pode ser analisado pela tomada de decisão (Romeas et al., 2016). Os resultados do presente estudo corroboram o modelo teórico de Martens et al. (1990), visto que a ansiedade cognitiva se relacionou negativamente com a tomada de decisão do saque, da recepção e do levantamento. Cabe destacar que a ansiedade cognitiva explicou, $28 \%, 19 \%$ e $21 \%$ da variância da tomada de decisão do saque, da recepção e do levantamento, respectivamente. Neste sentido, é provável que as expectativas negativas sobre o desempenho afete negativamente algum dos componentes da tomada de decisão: percepção, atenção e memória de trabalho. Segundo Fernandes et al. (2013), pensamentos negativos concernentes ao desempenho esportivo podem reduzir a capacidade de manutenção do foco de atenção, bem como a capacidade de processamento de informação do ambiente, o que, de certo modo, pode explicar os achados da ansiedade cognitiva na tomada de decisão de jovens atletas de voleibol na presente pesquisa.

A ansiedade somática, por sua vez, de acordo com o modelo teórico de Martens et al. (1990), possui relação curve-linear (U invertido) com o desempenho esportivo. Mais especificamente, pode-se dizer que existe uma magnitude ideal de ansiedade somática (zona individual) para se obter a excelência esportiva. Caso a ansiedade somática seja baixa ou muito elevada, possivelmente o atleta não obterá bom desempenho competitivo. Concernente ao desempenho do aspecto cognitivo nos esportes coletivos (tomada de decisão), os resultados do presente estudo indicaram relação negativa com a ansiedade somática. Ademais, os modelos de regressão revelaram que a ansiedade somática explicou $23 \%$, $26 \%$ e $18 \%$ da variância da tomada de decisão do saque, da recepção e do levantamento, respectivamente. Talvez, o aumento da frequência cardíaca, a sudorese elevada e o aumento da temperatura corporal, os quais são controlados pelo sistema nervoso autônomico (Fernandes et al., 2013), de alguma forma, podem inibir a percepção, o foco de atenção ou o acesso a memória de longo prazo.

Estudos têm apontado que o aumento do estresse psicofisiológico induzido pelo sistema nervoso autônomo faz com que a velocidade de transmissão de impulsos nervosos seja atenuada (Andersen \& Calatayud, 2016; Zhang, Si, Chung, \& Gucciardi, 2016), explicando parcialmente os resultados da presente investigação. Acrescentando, vale ressaltar que algumas respostas da ansiedade somática, tais como a descarga adrenérgica, a sobrecarga cardiovascular e a tensão muscular, podem reduzir a capacidade de processamento de informação dos lobos parietal e frontal (Bray, Graham, \& Saville, 2014), os quais controlam as etapas do processo de tomada de decisão (Romeas et al., 2016), o que, cautelosamente, pode ser uma explicação para os resultados concernentes a ansiedade somática na presente pesquisa. Em síntese, o famoso "nervosismo" momentos antes de uma partida decisiva possivelmente gera desempenho não desejável em jovens atletas de voleibol do sexo masculino.

No que diz respeito a autoconfiança, o modelo teórico de Martens et al. (1990) aponta relação linear e positiva com o desempenho esportivo, corroborando os achados do presente estudo. Em adição, os resultados revelaram que a autoconfiança respondeu, respectivamente, $18 \%, 11 \%$ e $23 \%$ da variância da tomada de decisão do saque, da recepção e do levantamento. $\mathrm{O}$ estudo de revisão sistemática realizado por Patel et al. (2010) revelou que atletas com nível de autoconfiança elevado obtiveram melhores resultados esportivos. No mesmo sentido, Fernandes et al. (2014) demonstraram que a autoconfiança esteve intimamente 
relacionada ao desempenho de atletas amadores. Assim, parece que o nível de autoconfiança do atleta pode impactar positivamente no seu desempenho competitivo. Ressalta-se, todavia, que nenhuma das duas investigações mencionadas acima (Patel et al., 2010; Rathschlag \& Memmert, 2014) analisou a relação da autoconfiança com o desempenho cognitivo. De nosso conhecimento, o presente estudo foi o primeiro que buscou avaliar o efeito da autoconfiança sobre a tomada de decisão de jovens atletas de voleibol, o que torna inviável a comparação com a literatura científica. Logo, os achados devem ser interpretados com cautela.

Embora o desenho experimental da presente pesquisa seja inédito com jovens atletas de voleibol, os resultados devem ser analisados com cuidado, visto que a investigação é dotada de limitações, especialmente quanto à não utilização de ferramenta para aferição de indicadores fisiológicos de ansiedade somática (e.g., frequência cardíaca), bem como não ter controlado os gestos técnicos na execução motora das ações de jogo. Salienta-se também a impossibilidade de generalização dos resultados para a população de jovens atletas de voleibol do sexo masculino, considerando que o tamanho amostral foi relativamente pequeno.

Os resultados do presente estudo permitiram concluir que a ansiedade pode ser considerada um fator interveniente na tomada de decisão de jovens atletas de voleibol do sexo masculino. Ressalta-se que os pensamentos negativos e os elementos fisiológicos da ansiedade afetaram negativamente a tomada de decisão, enquanto a convicção e o sentimento de capacidade afetou positivamente a tomada de decisão. Do ponto de vista prático, a presente pesquisa indica que a ansiedade cognitiva e somática pode impedir, ao passo que a autoconfiança pode permitir que o jovem atleta de voleibol realize tomada de decisão apropriada no decorrer de uma competição. Por fim, recomenda-se a realização de futuras investigações que busquem analisar o efeito da ansiedade competitiva (cognitiva, somática e autoconfiança) na tomada de decisão em jovens atletas de voleibol do sexo masculino em diferentes níveis competitivos (dificuldade da partida).

\section{REFERÊNCIAS}

Afonso, J., Garganta, J., \& Mesquita, I. (2012). A tomada de decisão no desporto: o papel da atenção, da antecipação e da memória. Revista Brasileira de Cineantropometria e Desempenho Humano, 14(5), 592-601. doi: 10.5007/1980-0037.2012v14n5p592

Andersen, L. L., \& Calatayund, J. (2016). Mind-muscle connection revisited: Do 100 studies about beanbag tossing, stick balancing, and dart throwing have any relevance for strength training? European Journal of Applied Physiology, 116, 865-866. doi: 10.1007/s00421-016-3342-x

Araújo, D., Davids, K., Diniz, A., Rocha, L., Santos, J. C., Dias, C., \& Fernandes, O. (2015). Ecological dynamics of continuous and categorical decision-making: The regatta start in sailing. European Journal of Sport Science, 15(3), 195-202. doi: 10.1080/17461391.2014.928749

Bangsbo, J. (2015). Performance in sports - With specific emphasis on the effect of intensified training. Scandinavian Journal of Medicine \& Science in Sports, 25(sup 4), 88-99. doi: 10.1111/ sms. 12605

Bray, S. R., Graham, J. D., \& Saville, P. D. (2014). Self-control training leads to enhanced cardiovascular exercise performance. Journal of Sports Sciences. doi: 10.1080/02640414.2014.949830

Costa, G. D. T., Ceccato, J. S., Evangelista, B. F. B., Freire A. B., Oliveira A. S., Milistetd M., Rodrigues, H. A., \& Ugrinowitsch, H. (2016). Tactic determinants of game practiced by middle attacker in men's volleyball. Revista Brasileira de Cineantropometria \& Desempenho Humano, 18(3), 371-379. doi: 10.5007/1980-0037.2016v18n3p371

Davids, K., Araújo, D., Correia, V., \& Vilar, L. (2013). How smallsided and conditioned games enhance acquisition of movement and decision-making skills. Exercise and Sport Sciences Reviews, 41(3), 154-161.

Fernandes, M. G., Nunes, A. S., Raposo-Vasconcelos, J., Fernandes, H. M., \& Brustad, R. (2013). The CSAI-2: An examination of the instrument's factorial validity and reliability of the intensity, direction and frequency dimensions with Brazilian athletes. Journal of Applied Sport Psychology, 25(4), 377-391. doi: 10.1080/10413200.2012.744780

Fernandes, M. G., Nunes, A. S., Raposo-Vasconcelos, J., \& Fernandes, H. M. (2014). Efeitos da experiência nas dimensões de intensidade, direção e frequência da ansiedade e autoconfiança competitiva: Um estudo em atletas de desportos individuais e coletivos. Motricidade, 10(2), 81-89. doi: 10.6063/motricidade.10(2).2930

Fernandes, M. G., Raposo-Vasconcelos, J., \& Fernandes, H. M. (2012) Propriedades psicométricas do CSAI-2 em atletas Brasileiros. Psicologia: Reflexão e Crítica, 25(4), 679-687. doi: 10.1590/S0102-79722012000400007

Fortes, L. S. Costa, B. D. V., Paes, P. P., Nascimento-Júnior, J. R. A., Vieira, L. F., \& Ferreira, M. E. C. (2017). Influence of competitive-anxiety on heart rate variability in swimmers. Journal of Sports Science and Medicine, 16(4), 498-504.

Fortes, L. S., Freitas-Júnior, C. G., Paes, P. P., Vieira, L. F., Nascimento-Júnior, J. R. A., Lima-Júnior, D. R. A. A., \& Ferreira, M. E. C. (2018): Effect of an eight-week imagery training programme on passing decision-making of young volleyball players. International Journal of Sport and Exercise Psychology. doi: 10.1080/1612197X.2018.1462229

Fortes, L. S., Lira, H. A. A. S., Lima, R. C. P., Almeida, S. S., \& Ferreira, M. E. C. (2016). Mental training generates positive effect on competitive anxiety of young swimmers? Revista Brasileira de Cineantropometria e Desempenho Humano, 18(3), 353-361. doi: 10.5007/1980-0037.2016v18n3p353

Kinrade, N. P., Jackson, R. C., \& Ashford, K. J. (2015). Reinvestment, task complexity and decision making under pressure in basketball. Psychology of Sport and Exercise, 20, 11-19. doi: 10.1016/j.psychsport.2015.03.007

Lopes, M. C., Magalhães, R. T., Diniz L. B. F., Moreira J. P. A., \& Albuquerque, M. R. (2016). The influence of technical skills on decision making of novice volleyball players. Revista Brasileira de Cineantropometria \& Desempenho Humano, 18(3), 362-370. doi: 10.5007/1980-0037.2016v18n3p362

Martens, R., Vealey, R. S., \& Burton, D. (1990). Competitive anxiety in sport. Champaign, IL: Human Kinetics.

Matias, C. J. A. S., \& Greco, P. J. (2011). Conhecimento táticoestratégico dos levantadores brasileiros campeões de voleibol: Da formação ao alto nível. Revista Brasileira de Educação Física e Esporte, 25(3), 513-35. doi: 10.1590/S1807-55092011000300014

Memmert, D., \& Harvey, S. (2008). The Game Performance Assessment Instrument (GPAI): Some concerns and solutions 
for further development. Journal of Teaching in Physical Education, 27, 220-240.

Millet, G. P., Groslambert, A., Barbier, B., Rouillon, J. D., \& Candau, R. B. (2005). Modelling the relationships between training, anxiety, and fatigue in elite athletes. International Journal of Sports Medicine, 26(6), 492-498.

Oslin, J. L., Mitchell, S. A., \& Griffin, L. L. (1998). The game performance assessment instrument (GPAI): Development and preliminary validation. Journal of Teaching in Physical Education, 17, 231-243.

Patel, D. R., Omar, H., Terry, M. (2010). Sport-related performance anxiety in young female athletes. Pediatric Adolescence Gynecology, 23(3), 325-335. doi: 10.1016/j.jpag.2010.04.004

Rathschlag, M., \& Memmert, D. (2015). Self-generated emotions and their influence on sprint performance: An investigation of happiness and anxiety. Journal of Applied Sport Psychology, 27, 186-199. doi: 10.1080/10413200.2014.974783
Romeas, T., Guldner, A., \& Faubert, J. (2016). 3D-Multiple Object Tracking training task improves passing decision-making accuracy in soccer players. Psychology of Sport and Exercise, 22, 1-9. doi: 10.1016/j.psychsport.2015.06.002

Silva, M. M. F., Vidual, M. B. P., Oliveira, R. A., Yoshida, H. M., Borin, J. P., \& Fernandes, P. T. (2014). Ansiedade e desempenho de jogadoras de voleibol em partidas de voleibol realizadas dentro e fora de casa. Revista da Educação Física/UEM, 25(4), 585-596. doi: 10.4025/reveducfis.v25i4.24347

Sonoo, C. N., Gomes, A. L., Damasceno, M. L., Silva, S. R., \& Limana, M. D. (2010). Ansiedade e desempenho: Um estudo com uma equipe infantil de voleibol feminino. Motriz, 16(3), 629-637. doi: 10.5016/1980-6574.2010v16n3p629

Zhang, G., Si, P., Chung, P., \& Gucciardi, G. F. (2016). Mindfulness and burnout in elite junior athletes: The mediating role of experiential avoidance. Journal of Applied Sport Psychology, 20,1-15. doi: 10.1080/10413200.2016.1162223 